\title{
Influence of Low BMI on Handgrip Strength, Handgrip Endurance and Hand Dexterity in School Going Children- A Pilot Study
}

\author{
Padmanabhan Suresh Babu Roshan ${ }^{1}$, Chandrashekar ${ }^{2}$, Likhitha $\mathbf{N ~ J}^{3}$ \\ ${ }^{1}$ Assistant Professor, ${ }^{3}$ Post Graduate Student, Laxmi Memorial College of Physiotherapy, Mangalore, 575002 \\ ${ }^{2}$ Associate Professor, Department of Paediatrics, A.J Institute of Medical Science \& Research Centre, \\ Mangalore \\ Corresponding Author: Likhitha N J
}

\begin{abstract}
Introduction: The hand is the most dynamic and interactive part of the upper limb in humans and performing the complex tasks of daily living activities. The health problems due to less-thanoptimal nutritional status in primary school-age children are among the most common causes of low school enrolment, high absenteeism, early dropout, and unsatisfactory classroom performance.
\end{abstract}

Purpose: To compare handgrip strength, handgrip endurance, and hand dexterity in 6-12 years children with low and normal body mass index.

Methodology: This pilot study was conducted among children with normal development. The age group included in the study was 6-12 years. The estimated sample 25 in each group. Participants underwent initial assessment, where height and weight were measured. To measure handgrip strength and handgrip endurance baseline hydraulic hand dynamometer and hand dexterity were assessed using a 9-hole pegboard, the participants were asked to perform three successful trials, and the mean of it was obtained.

Results: Result shows that there was a significant difference between handgrip strength with BMI, with $\mathrm{p}<0.01$ and no significant difference between handgrip endurance and hand dexterity with $p>0.05$ in children.

Conclusion: The results of the current study demonstrated that 6-12-year-old children had a significant difference in handgrip strength of the dominant and non-dominant hand between low BMI and normal BMI children. With further research, it is important to find and compare the differences in a larger population.

Keywords: Body mass index, dominant hand, handgrip strength, handgrip endurance, hand dexterity, non-dominant hand.

\section{INTRODUCTION}

The body mass index (BMI) is the most basic and acceptable technique for evaluating relative body fatness. Body mass index (BMI) usually defines body fatness as an index of weight relative to height and is generally considered a valid index of adiposity. ${ }^{[1]}$ Although body mass index (BMI) is a proxy measure of body fat since it assesses excess weight rather than excess fat and is frequently used as an indicator of body fatness. High body mass index (BMI) predicts future morbidity and death. Therefore, body mass index (BMI) is an appropriate measure for screening for obesity and its health risks. ${ }^{[1]}$

The national family health survey (NFHS) data shows that $53 \%$ of children in rural areas are underweight or have low weight for age and this varies across states. The percentage of underweight children in the country was 53.4 in 1992 , it decreased to 45.8 in 1998 and rise again to 47 in $2006 .^{[2]}$ According to a UNICEF report of 2019, about 33 percent of children in India are suffering from underweight and 2 percent of total children in India are suffering from overweight. At the same time, the Indo- 
Asian countries are facing a unique challenge of growing overweight/obesity and a persistent burden of undernutrition. ${ }^{[3]}$

The young population is the backbone of a nation only because they are usually presented with better activity levels and lifestyle characteristics. In daily living, the major proportion of usage of hand involves the dominant hand. Many tasks of everyday living and sports performance necessitate a high level of activity in the muscles of the hands and forearms. These muscles are required for the execution of some skills involving physical demands and endurance. ${ }^{[4]}$ Children's muscular strength is associated with overall health, bone health, and self-esteem and inversely associated with metabolic and cardiovascular disease biomarkers. ${ }^{[5]}$

The human hand is a complex structure tailored to the functions of manipulation, and the hand conveys sensory information to the brain about the temperature, and texture of the objects it controls. ${ }^{[6]}$ Work activities, as well as play and leisure activities, require both grip strength and manual dexterity. Moreover, $60 \%$ of school activities require fine motor and manual dexterity skills. ${ }^{[7]}$ Skilled hand use involves the ability for one to have individual control over their fingers, a somatosensory system capable of guiding hand motions, and the ability to respond to sensory input with a suitable hand configuration. When children struggle with any of these skills, difficulties might arise in routine daily actions such as picking up small things between the thumb and index fingers, when playing with little toys and handling buttons and zippers, when dressing as children develop and continue to experience difficulties with fine motor tasks, their overall independence and ability to participate in the activities of daily living and school become further limited. ${ }^{[8]}$

The handgrip is an important component of human function and is a unique feature that distinguishes humans from primates. ${ }^{[9]}$ Handgrip strength (HGS) is a well-established indicator of muscle strength and can help to identify the risk of sarcopenic obesity in children. ${ }^{[10]}$ Handgrip strength (HGS) is essential to many sports such as rock climbing, wrestling, handball, tennis, swimming, and so on, and it is thus regarded as a factor in maximizing performance and control. ${ }^{[4]}$ The power of the handgrip is the result of forceful flexion of all finger joints with the subject's maximum voluntary force under normal biokinetic conditions. Hand strength is an important independent surrogate parameter to assess outcome and risk of morbidity and mortality. ${ }^{[11-14]}$ Handgrip is an important component of human function and is a unique feature that distinguishes humans from primates. ${ }^{[9]}$ Various factors affect handgrip strength among children, including age, height, weight, bone density, muscle mass, posture, hand dominance, forearm girth, etc. ${ }^{[4,12,15-17]}$

Handgrip strength (HGS) and handgrip endurance (HGE) are important parameters to assess the upper extremity muscular strength of an individual and play a pivotal role in the prevention of injuries, especially during sports activities. [16,18] Muscular endurance is the ability of a muscle or muscle group to perform against a load for an extended period of time, measured in seconds. ${ }^{[19]}$ Endurance of the ability of a muscle to endure the power produced during activity is referred to as its strength. In other words, Handgrip endurance (HGE) is the ability to sustain a muscular force. It has often been used as a measure of physical performance. ${ }^{[16]}$

Dexterity is generally defined as the coordination of small muscle movements usually involving the synchronization of hands and fingers with the eyes. ${ }^{[20]}$ Manual dexterity (hand function) is an individual's ability to coordinate the fingers and manipulate objects in a timely manner. ${ }^{[21]}$ Handgrip strength (HGS) and pinch strength (PS) are determinative for performing prehensile and precision hand functions and daily muscular activities through the use of exquisitely arranged power and precision muscles, which acting through the extensor 
hood, work in synergistic precision to manipulate the digits. ${ }^{[22]} \mathrm{A}$ dexterity test is designed so that it provides information about the quality and speed of performance of the hand while the subject accomplishes a task, making it easier for the examiner to quantify the quality of performance and manipulate the resulting data for deeper analysis. ${ }^{[20]}$ Dexterity is an important component of evaluating hand function. These skills are important in adults, but even more so in children where the development of dexterity plays a large role in their functional and physical development. $^{\text {[23] }}$

Handgrip strength, handgrip endurance, and hand dexterity are important in school-going children because most of the skilled activities of children during school time depend on the activities of hand. If BMI is low, the child may not have adequate strength in hand when compared to children with normal BMI. This will affect the physical activity in children and children may lag in every activity in the school with peer groups. So, the aim of the study is to address the influence of low BMI on handgrip strength, handgrip endurance, and hand dexterity in school-going children when compared to normal BMI.

\section{MATERIALS AND METHODS}

This pilot study was carried out in a period of 6 months. Children aged 6-12 years were included from schools in Karnataka, were screened for inclusion and exclusion criteria, after seeking permission from the Principals of the respected schools, the subjects falling within the inclusion criteria were recruited for the study. A sample size of 25 low BMI and 25 normal BMI was estimated. A purposive sampling technique was used to include the participants in the study. Ethical clearance was obtained from the Institutional ethical committee. The parents of the subjects participating in the study were given an information sheet containing the study details and, also the child assent consent form was obtained from the subjects prior to the study. Inclusion criteria for the study were 6-12 years of age group, with normal development, children with low BMI and normal BMI, participating in normal daily activities. Exclusion criteria were those with any unhealed injury in hand, those with a history of an upper limb fracture in the past 2 months, children with previous hand surgery, deformities on the upper arm, any neuromuscular disorder or system pathology that affects the hand function, all diagnostic conditions and surgical histories were identified through parent's report.

Materials: Hand-held dynamometer, 9-hole pegboard, Stopwatch, Stadiometer, Standardized weighing machine, Table and chair, Pen, paper.

Procedure: At first, informed consent was obtained from the parents of the participants. A detailed procedure was explained about the test to all participants. All the recruitment of the participants in this study was held according to the inclusion and exclusion criteria. After the recruitment, an initial examination did include the following: demographic data, height, weight, handgrip strength, handgrip endurance, hand dexterity.

Outcome measures: To measure the handgrip strength and handgrip endurance: handheld dynamometer. To assess hand dexterity: 9-hole pegboard.

\section{Body mass index (BMI): Quetelet's index}

Is a value derived from the mass (weight) and height of an individual. Each participant's height was measured to the nearest centimeter with a stadiometer barefoot and weight was measured kilogram by a standard weighing machine. Then body mass index (BMI) was calculated by using Quetelet's index (body mass divided by the square of the body height and it is expressed in units of $\mathrm{kg} / \mathrm{m}^{2}$ ). According to the world health organization (WHO) classification of body mass index (BMI), participants were categorized into two groups as underweight $<15 \mathrm{~kg} / \mathrm{m}^{2}$ (below the 5th percentile) and 
normal weight $\quad 15-22 \mathrm{~kg} / \mathrm{m}^{2} \quad$ (5th-84th percentile). ${ }^{[24-28]}$

\section{Handgrip strength and endurance: Handheld dynamometer test}

The handgrip strength and endurance of the dominant hand and nondominant hand were measured by using a standard handgrip dynamometer (Baseline Hydraulic hand dynamometer). Prior to the test, each participant was given verbal instruction and demonstration of the test. Participants were in a sitting position with shoulder adducted and neutrally rotated, elbow at $90^{\circ}$ flexion, forearm, and wrist in a neutral position. Participants were instructed to squeeze the handle of the dynamometer as hard as they could and to sustain the effort for 5 seconds and verbal encouragement was provided to children during testing. Each participant performed 3 trials for each hand, and the mean values of these trials were recorded in kilograms (kg). Participants were given 1 minute to rest between trials, and trials were completed with alternating hands to minimize the effects of fatigue and a brief pause of 20-30 secs were given between each trial. Handgrip endurance (HGE) was determined by asking the subject to sustain 1/3rd of maximal voluntary contraction (MVC) for as long as he/she could. The subject was made to sit with the forearm placed on the edge of the table followed by elbow flexed at 900 , forearm mid-prone position and was asked to maintain a grip of $1 / 3$ rd of MVC and then the time recording was noted in seconds by using a stopwatch. ${ }^{\text {22,29-30] }}$

\section{To assess hand dexterity: 9-Hole pegboard test}

Hand dexterity was measured using the 9-hole pegboard test. The 9-hole pegboard is a 5- inch square pegboard. Each peg hole is $3 \mathrm{~cm}$ deep, $2.5 \mathrm{~cm}$ in diameter, and $2 \mathrm{~cm}$ apart from the next peg hole in a row of three. Each peg is $4 \mathrm{~cm}$ tall and 2.2 $\mathrm{cm}$ in diameter, with a dot marked on one side. The 9-HPT was timed using a stopwatch. Each participant was tested separately while he/she sits at a desk and chair of appropriate height with their feet supported on the floor to ensure that the tabletop is at the mid-chest level. The pegboard is centered in front of a participant with the container next to the board on the same side of the hand being tested. First, the dominant hand is tested, followed by the non-dominant hand. The participant is instructed to pick up 1 peg at a time using 1 hand and to put them in the holes until all 9 were filled. The participant then removed the pegs from the holes one by one. The order of placement is not prescribed. For the non-dominant hand, the pegboard is turned so that the container is on the same side of the non-dominant hand. During the test, the therapist instructed the participant not to touch the peg with the free hand and scoring was a total time (seconds) to complete the task. Timing began on contact with the first peg and ended with the return of the final peg to the board. The average time of 3 trials was used in the analysis. ${ }^{\text {22,31,32] }}$

\section{Statistical Methods}

The collected data were summarized using descriptive statistics such as mean+ S.D. (standard deviation) to find the difference between body mass index with handgrip strength, handgrip endurance, and hand dexterity in 6-12 years old children. An independent sample t-test was used, pvalue $<0.01$ was considered as significant. The data was analyzed using Microsoft Excel and SPSS 21. The obtained p-value is $>0.05$ hence there is no significant difference in children.

\section{RESULTS}

Table 1: Descriptive statistics on the age-wise distribution of the study participants $(n=50)$

\begin{tabular}{|c|c|c|c|c|c|c|}
\hline & N & Range & Minimum & Maximum & Mean & Std. Deviation \\
\hline Age & 50 & 6.00 & 6.00 & 12.00 & 9.7400 & 2.04849 \\
\hline
\end{tabular}


Among 50 individuals included in the study, it was observed that the mean age is 9.74 years with the standard deviation of 2.04 years.

Pie chart representing the genderwise distribution of the study participants

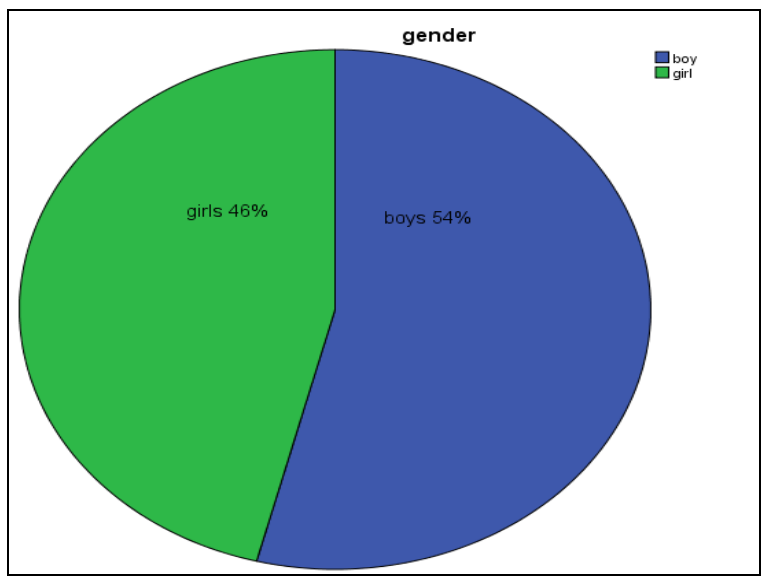

Figure1: Majority of the study participants are boys (46\%).

Table 2: Distribution of handgrip strength, handgrip endurance, hand dexterity, Mean + S.D. (Standard Deviation) and p value

\begin{tabular}{|l|l|c|c|c|}
\hline & & Mean & S. D & p value \\
\hline BMI & & 15.56 & 2.04 & \\
\hline Dominant HGS & Low BMI & 10.47 & 2.82 & .001 \\
\hline & Normal BMI & 13.39 & 3.07 & \\
\hline Dominant HE & Low BMI & 54.40 & 29.11 & .041 \\
\hline Dominant HD & Normal BMI & 40.64 & 15.02 & \\
\hline & Low BMI & 30.56 & 4.43 & .597 \\
\hline Non dominant HGS & Normal BMI & 31.26 & 4.84 & \\
\hline & Low BMI & 9.65 & 2.66 & .002 \\
\hline Non dominant HGE & Normal BMI & 12.23 & 3.02 & \\
\hline & Low BMI & 35.40 & 23.34 & .379 \\
\hline Non dominant HD & Normal BMI & 30.24 & 17.33 & \\
\hline & Low BMI & 32.71 & 4.05 & .454 \\
\hline & Normal BMI & 31.75 & 4.88 & \\
\hline
\end{tabular}

An independent sample t-test was calculated between the parameters. It shows that there is a significant difference between BMI with handgrip strength of dominant and non-dominant hand $(\mathrm{p}<0.01)$.

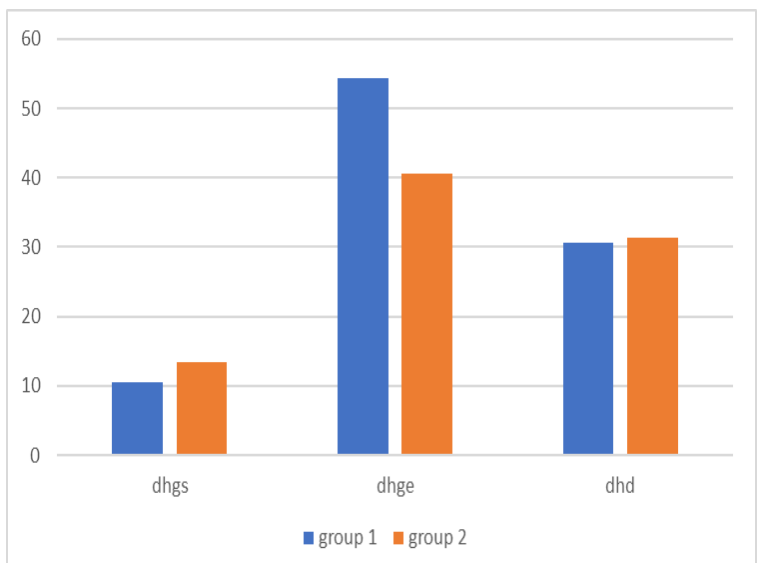

Figure2: Multiple Bar diagram representing the handgrip strength, handgrip endurance, and hand dexterity of the dominant hand
A significant different was found between BMI and HGS of dominant hand with low BMI and normal BMI $(\mathrm{p}<0.01)$ and no significant different was found between BMI and HGE, HD of dominant hand with low BMI and normal $\mathrm{BMI}(\mathrm{p}>0.05)$ in children.

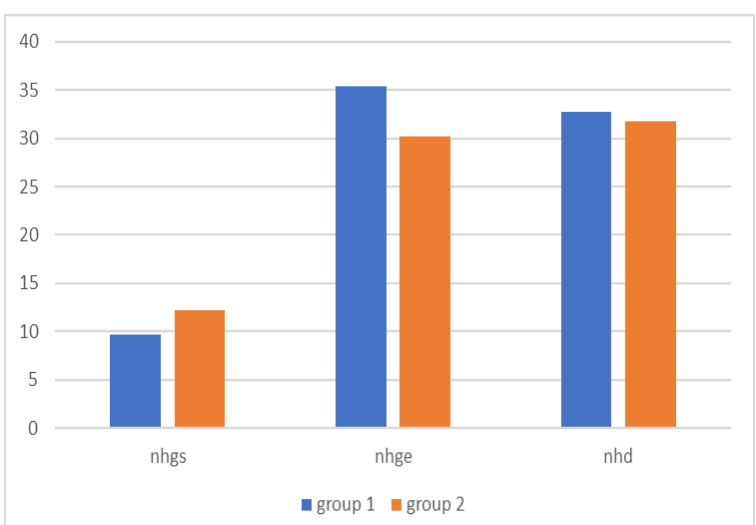

Figure3: Multiple Bar diagram representing the handgrip strength, handgrip endurance and hand dexterity of the non-dominant hand

A significant different was found between BMI and HGS of non-dominant hand with low BMI and normal BMI $(\mathrm{p}<0.01)$ and no significant different was found between BMI and HGE, HD of nondominant hand with low BMI and normal BMI ( $p>0.05)$ in children.

\section{DISCUSSION}

A variety of daily functions and sporting events necessitate a high amount of flexor muscle activity in the forearms and hands. There are 35 muscles involved in forearm and hand movement, with several of them involved in grasping tasks. Factors such as muscular strength, hand dominance, fatigue, time of day, age, gender, nutritional status, restricted motion, and pain always have an impact on grip strength. Handgrip strength has also been related to nutritional status. ${ }^{[32]}$

Physiological hand shape indicators are predictors of children's physical growth and development, and they are simple and easy to test. These markers are not only related to children's grip strength, pinch strength, height, and weight, but also to psychological and behavioral difficulties, fine movement, and writing skills. Several 
studies have demonstrated that physiological hand shape indicators increase with age, with boys having larger physiological hand shape indicators than girls of the same age in most age groups. ${ }^{[33]}$ Hands and feet typically develop faster than other parts of the body. Therefore, handgrip strength is considered to be an important indicator of children's developmental level. ${ }^{[15]}$

This observational study was designed to evaluate the difference between low body mass index (BMI) handgrip strength (HGS), handgrip endurance (HGE) and hand dexterity with normal body mass index (BMI) handgrip strength (HGS), handgrip endurance (HGE) and hand dexterity in school-going children.6-12 years old children schools in Karnataka were recruited on the basis of inclusion and exclusion criteria. A total number of 50 children; 25 low body mass index (BMI) and 25 normal body mass index (BMI) children were selected and an explanation about the procedure was given. The demographical data including age, height, and weight were recorded.

Descriptive statistics were used to find out the frequency, percentage, mean and standard deviation from demographic data and variables studied. This mean age was 9.74 years with a standard deviation of 2.04 years. The maximum age and minimum age were 12 and 6years. $32.0 \%$ of children were in the age group of 12 years, $26.0 \%$ in the age group of 10 years, $12.0 \%$ of children were in the group of 9 years, $10.0 \%$ in the age group of 6 and 7 years, $6.0 \%$ in 8 years, and $4.0 \%$ in 11 years. And with a minimum BMI of $12.98 \mathrm{~kg} / \mathrm{m}^{2}$, a maximum BMI of $20.54 \mathrm{~kg} / \mathrm{m}^{2}$ with a mean of $15.56 \mathrm{~kg} / \mathrm{m}^{2}$, and a standard deviation of $2.02 \mathrm{~kg} / \mathrm{m} 2$. The majority of the study population are boys (54\%) than girls (46\%).

Hand-held dynamometer tests were used to find out the handgrip strength and handgrip endurance in children, 9-hole pegboard test was carried out for hand dexterity in children with low body mass index and normal body mass index in both dominant hand and non-dominant hand.

After analyzing data, using $\mathrm{Z}$ test, dominant hand and non-dominant hand low BMI and normal BMI, HGS statistically significant difference were observed

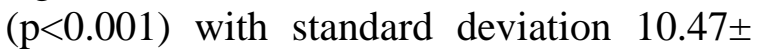
2.82 and $13.39 \pm 3.07,9.65 \pm 2.66$ and $12.23 \pm$ 3.02 respectively, $\mathrm{HGE}$ and $\mathrm{HD}$ of the dominant hand and non-dominant hand low BMI and normal BMI, statistically no significant difference was observed $(\mathrm{p}>0.05)$ with standard deviation $54.40 \pm 29.11$ and $40.64 \pm 15.02,35.40 \pm 23.34$ and $30.24 \pm 17.33$, $30.56 \pm 4.43$ and $31.26 \pm 4.84,32.71 \pm 4.05$ and $31.75 \pm 4.88$ respectively.

But the result of this study showed that handgrip strength with BMI of dominant and non-dominant hand has a strong significant relation with $\mathrm{p}<0.01$ and handgrip endurance and hand dexterity with BMI of the dominant hand and nondominant hand have no significant relation with $\mathrm{p}>0.05$.

There are some studies that show a negative correlation between BMI and HGS and some studies show a positive correlation between BMI and HGE. Bohannon RW et al [31] concluded that as moderate to strong relationships between grip strength and dexterity and fine hand use are attenuated by age in children of 3 to 13 years, they cannot recommend the use of anyone measure over others to characterize the motor function of the hand. Mohammed T.A. et al ${ }^{[22]}$ concluded that gender, age, height, and weight are positively associated with the development of grip strength and dexterity function in children.

Incel NA et al concluded that the dominant hand is significantly stronger in right-handed subjects but no such significant difference between sides could be documented for left-handed people. This difference may be attributed to the fact that left-handed people are temporarily forced to use their non-dominant hands for daily activities in this right-hand dominant world. $^{[34]}$ 
Sanyukta Gurung L et al concluded that a significant correlation of BMI with handgrip strength and handgrip endurance was seen in this study. Male participants had better handgrip strengths whereas female participants had better handgrip endurance. A negative correlation was seen between BMI and handgrip strength in the underweight and overweight categories. A positive correlation was observed between handgrip endurance and all the BMI categories in both male and female participants. $^{[18]}$

The study has the implication of, the estimation of handgrip strength, handgrip endurance, and hand dexterity is important in determining the efficacy of different treatment strategies of the hand and also in hand rehabilitation. It is often used as an indicator of general physical strength, the performance of the hand and forearm muscles, and as a functional index of nutritional condition. In order to properly diagnose various musculoskeletal deformities, especially related to upper extremities

We cannot completely rely on this information because this study was conducted in a small population, which cannot be considered as the representation of the whole population. Hence the suggestion is that there should be further studies on the same population factor in children with normal development. Future investigations should include a large sample size. There are various limitations in this study was small sample sizes and children from a particular school were recruited in this study. A study with a larger sample size which is inclusive of different age groups and hand dominance and also subjects from different socioeconomic statuses and wide geopolitical areas is advocated to come to a conclusion. Due to these limitations, the results of this study require further investigation to be conducted.

\section{CONCLUSION}

The results of the current study demonstrated that 6-12-year-old children had a significant difference in handgrip strength of the dominant and non-dominant hand between low BMI and normal BMI children. With further research, it is important to find and compare the differences in a larger population.

\section{Acknowledgement: None}

Conflict of Interest: None

\section{Source of Funding: None}

\section{Ethical Approval: Approved}

\section{REFERENCES}

1. Shivakumar S, Vileep KS. Study on BMI status of rural high school boys among various academic divisions of Karnataka state. IJYPPE. 2018; 3(1); 49-51

2. International Institute of Population Sciences (IIPS) National Family Health Survey (NFHS-3), Fact sheets for 29 States. Mumbai: International Institute for Population Sciences India, Mumbai 2007.

3. The State of the World's Children 2019 [Internet]. Unicef.org. 2021 [cited 23 June 2021]. Available from: https://www.unicef.org/reports/state-ofworlds-children-2019.

4. Hammed A, Obaseki C. Interdependence of body mass index with handgrip strength and endurance among apparently healthy teenagers. Turk.j. kinesiol. 2018;4(1):1-7.

5. Garcia-Hermoso A, Cofre-Bolados C, Andrade-Schnettler R, Ceballos-Ceballos R, Fernández-Vergara $\mathrm{O}$, Vegas-Heredia $\mathrm{E}$ et al. Normative Reference Values for Handgrip Strength in Chilean Children at 812 Years Old Using the Empirical Distribution and the Lambda, Mu, and Sigma Statistical Methods. J strength cond. res2021;35(1):260-266.

6. Tremblay J, Curatolo S, Leblanc M, Patulli C, Tang T, Darsaklis V et al. Establishing normative data for the functional dexterity test in typically developing children aged 35 years. J Hand Ther.2019;32(1):93-102.e2.

7. Angst F, Drerup S, Werle S, Herren D, Simmen B, Goldhahn J. Prediction of grip and key pinch strength in 978 healthy subjects. BMC Musculoskelet. Disord. 2010;11(1). 
8. Jürimäe T, Hurbo T, Jürimäe J. Relationship of handgrip strength with anthropometric and body composition variables in prepubertal children. HOMO. 2009;60(3): 225-238.

9. Abdullahi Y, Audu T, Goon D. Handgrip strength and body mass index among adolescents in Northern Nigeria. J. HEALTH Sci2020;

10. Massy-Westropp N, Gill T, Taylor A, Bohannon R, Hill C. Handgrip strength: age and gender stratified normative data in a population-based study. BMC Res.Notes 2011;4(1).

11. Chhaya Verma1 P. Study of Handgrip strength in Indian Population. Int. J. Health Sci2016;6(11):162-166.

12. Palacio-Agüero A, Díaz-Torrente $X$, Quintiliano Scarpelli Dourado D. Relative handgrip strength, nutritional status and abdominal obesity in Chilean adolescents. PLOS ONE. 2020;15(6): e0234316.

13. Hammed A, Agbonlahor E. Relationship between anthropometrics and handgrip strength among Nigerian school children. Biomed. Hum. Kinet.2017;9(1):51-56.

14. Dhananjaya JR, Veena HC, Mamatha BS, Sudarshan CR. Comparative study of body mass index, hand grip strength, and handgrip endurance in healthy individuals. Natl J Physiol Pharm Pharmacol 2017; 7(6):594.

15. Mahmoud A, Elhadidy E, Hamza M, Mohamed N. Determining correlations between handgrip strength and anthropometric measurements in preschool children. J. Taibah Univ. Medical Sci.2020; 15(1):75-81.

16. Manisha Jindal M. Relationship of HandGrip Strength and Endurance Time with an Individual's Anthropometric Parameters - A Study in Adolescent Population of Jammu. IJCMAAS 2017;14(3):119-123.

17. Koley S, Melton S. Age-related Changes in Handgrip Strength among Healthy Indian Males and Females Aged 6-25 years. J. Life Sci.2010;2(2):73-80.

18. Sanyukta Gurung L. Correlation of Body Mass Index with Handgrip Strength and Endurance of Dominant Hand in Medical Students. JIOM Nepal. 2020;42(3) (57-61).

19. Ibegbu A, Baita M, Hamman W, Emmanuel U, Musa S. Evaluation of the Relationship between Handgrip Strength with Some Anthropometries among Nigerian
Secondary School Students. The Anthropologist.2014;17(3):921-927.

20. Gonzalez V, Rowson J, Yoxall A. Development of the Variable Dexterity Test: Construction, reliability and validity. Int J Ther Rehabil 2015;22(4):174-180.

21. Wang Y, Bohannon R, Kapellusch J, Garg A, Gershon R. Dexterity as measured with the 9-Hole Peg Test (9-HPT) across the age span. $J$ Hand Ther.2015;28(1):53-60.

22. Omar M, Alghadir A, Zafar H, Al Baker S. Hand grip strength and dexterity function in children aged 6-12 years: A cross-sectional study. $J$ Hand Ther.2018;31(1):93-101.

23. Tissue C, Velleman P, Stegink-Jansen C, Aaron D, Winthrop B, Gogola G. Validity and reliability of the Functional Dexterity Test in children. $J$ Hand Ther.2017;30 (4): 500-506.

24. Srivastava A, Mahmood S, Srivastava P, Shrotriya V, Kumar B. Nutritional status of school-age children - A scenario of urban slums in India. Archives of Public Health. 2012;70(1):1-8.

25. Hammed A, Agbonlahor E. Relationship between anthropometrics and handgrip strength among Nigerian school children. Biomed. Hum. Kinet.2017;9(1):51-56.

26. Das A, Dutta M. correlation between body mass index and handgrip strength and handgrip endurance among young healthy adults. JEBMH. 2015;2(27):3995-4001.

27. Lakshmana swamy A. Clinical paediatrics, History taking and case discussion $4^{\text {th }}$ edition:12-14.

28. Santhosh Kumar A. Paediatric clinical examination $5^{\text {th }}$ edition: $15-16$.

29. Lee-Valkov PM, Aaron DH, Eladoumikdachi F, Thornby J, Netscher DT. Measuring normal hand dexterity values in normal 3-, 4-, and 5-year-old children and their relationship with grip and pinch strength. $J$ Hand Ther.2003 Jan 1;16(1):228.

30. Poole JL, Burtner PA, Torres TA, McMullen CK, Markham A, Marcum ML, et al Measuring dexterity in children using the Nine-hole Peg Test. $J$ Hand Ther.2005 Jul 1;18(3):348-51.

31. Bohannon RW, Wang YC, Noonan C. Relationships between grip strength, dexterity, and fine hand use are attenuated by age in children 3 to 13 years-of-age. $J$ Phys Ther Sci. 2019;31(4):382-6. 
Padmanabhan Suresh Babu Roshan et.al. Influence of low BMI on handgrip strength, handgrip endurance and hand dexterity in school going children- a pilot study

32. Häger-Ross C, Rösblad B. Norms for grip strength in children aged 4-16 years. Acta Paediatric. 2002 Jun;91(6):617-25.

33. Lad UP, Satyanarayana P, Shisode-Lad S, Siri CC, Kumari NR. A study on the correlation between the body mass index (BMI), the body fat percentage, the handgrip strength and the handgrip endurance in underweight, normal weight and overweight adolescents. JCDR. 2013 Jan;7(1):51.
34. Incel NA, Ceceli E, Durukan PB, Erdem HR, Yorgancioglu ZR. Grip strength: effect of hand dominance. SMJ 2002 May 1;43(5):234-7.

How to cite this article: Roshan PSB, Chandrashekar, Likhitha NJ. Influence of low BMI on handgrip strength, handgrip endurance and hand dexterity in school going children- a pilot study. International Journal of Science \& Healthcare Research. 2021; 6(3): 70-78. DOI: https://doi.org/10.52403/ijshr.20210713 